\title{
Modelos hipsométricos ajustados para um fragmento de cerrado Sensu stricto tocantinense
}

Igor Elói Silva Machado

Paulo Cesar Alves de Oliveira Medeiros²

Marla Guedes Cordeiro Carvalho 3

Claudia Alicia Marin Perez 4

Thiago Franco Santana 5

Valdir Carlos de Lima Andrade ${ }^{6}$

\section{Resumo}

Para uma redução dos custos de inventário florestal, os modelos hipsométricos são de grande valia. O presente trabalho teve como objetivo ajustar e avaliar modelos de relação hipsométrica em área de Cerrado em processo regenerativo situada no município de Gurupi (TO). Foram testados dez modelos, incluindo cinco lineares e cinco não lineares, no qual os ajustes foram realizados por meio do software R for Windows. Foram utilizados como critério de seleção do ajuste dos modelos os parâmetros estatísticos do coeficiente de determinação ajustado ( $\left.R^{2} a j.\right)$, o erro padrão residual $\left(\mathrm{s}_{\mathrm{HH}}\right)$ e a análise gráfica dos resíduos e, em seguida, a validação dos melhores modelos ajustados. As melhores estatísticas de ajuste foram para os modelos não lineares, com destaque para o modelo de Gompertz (modelo 7), R²aj: 0,489 e $\mathrm{s}_{\mathrm{H}}: 17,58 \%$. Os modelos, em geral, superestimaram a altura, atingindo até $40 \%$ a mais do que a altura das plantas reais. Diante disso, seis modelos foram para a validação, sendo dois lineares e quatro não lineares. 0 melhor modelo com a predição foi o Prodan não linear (modelo 10), que obteve melhores valores estatísticos e variação gráfica. Conclui-se que os modelos não lineares explicaram melhor a variável dependente altura.

Palavras-chave: Altura de árvores. Seleção. Validação.

\section{Introdução}

Com o panorama atual de reestruturação produtiva do capital, áreas do bioma Cerrado têm sido o foco em investimentos para implantação de fronteiras agrícolas (INOCÊNCIO, 2010). Sendo assim, é de suma importância que essa exploração ocorra de maneira sustentável, haja vista que, segundo o Instituto Brasileiro de Geografia e Estatística - IBGE (2004), o bioma Cerrado ocupa uma extensão de 23,9 \% do território brasileiro. No estado do Tocantins, esse bioma tem uma cobertura de 63,2 \% (SECRETARIA DO PLANEJAMENTO E MEIO AMBIENTE - SEPLAN, 2012).

Diante disso, em busca de uma exploração florestal baseada na sustentabilidade, é essencial ter o conhecimento das características da área a ser manejada, o que se consegue por meio de um

1 Universidade Federal do Tocantins, Mestrando em Ciências Florestais e Ambientais. igeloi@hotmail.com.

2 Universidade Federal do Tocantins, Mestrando em Ciências Florestais e Ambientais. paulocesar_machado@hotmail.com

3 Universidade Federal do Tocantins, Mestrando em Ciências Florestais e Ambientais. marla.guedes@hotmail.com.

4 Universidade Federal do Tocantins, Mestrando em Ciências Florestais e Ambientais. biologa.claudiamarin@gmail.com.

5 Universidade Federal do Tocantins, Mestrando em Ciências Florestais e Ambientais. mariathiagofranco@gmail.com.

6 Universidade Federal do Tocantins, Professor do Curso de Engenharia Florestal.vclandrade@uft.edu.br 
inventário florestal conduzido de forma eficaz, com baixo custo e alta confiabilidade. O inventário florestal aborda a definição quantitativa e qualitativa dos indivíduos em uma determinada área, sendo que a finalidade do inventário é especificada de acordo com o uso da área, que pode ser de reserva florestal, conservação da vida silvestre, reflorestamento comercial, área de lazer e outros (RODRIGUES; RODRIGUES, 2012).

Nos inventários, a medição do diâmetro das árvores é feita de forma prática e rápida, porém a altura das árvores é uma variável que necessita de mais tempo para mensuração, além da necessidade do emprego de instrumentos desenvolvidos para esse fim (hipsômetros), sendo alguns de alto investimento financeiro. Em situações adversas, sobretudo em matas nativas, que apresentam alta densidade e difícil visualização da copa e base da árvore, se torna difícil a medição das alturas das árvores (CRECHE, 1996; ANDRADE et al., 2006; SANQUETTA, 2013). Como opção para resolver esse problema, tem-se a utilização de modelos hipsométricos que permitem estimar a altura correlacionando-a com seu diâmetro à altura do peito (DAP) (SOARES et al., 2006).

Para uma redução dos custos de um inventário florestal, os modelos hipsométricos permitem que apenas algumas árvores tenham a sua altura medida nas parcelas e assim sejam ajustados modelos estatísticos dando mais rapidez nos levantamentos e tornando o inventário mais fácil de ser processado. Para se estimar uma variável dependente, neste caso a altura total das arvores, podem ser utilizados diversos modelos de regressão (SCHNEIDER et al., 2009), que podem ser tanto lineares quanto não lineares. Pontes Neto (2012) descreve que os modelos lineares são aqueles em que a sua estrutura se encontra na forma aditiva dos parâmetros. Modelos não lineares são aqueles em que os seus parâmetros são agregados de forma não aditiva e podem ser descritos como linearizáveis e não linearizáveis (DRAPER; SMITH, 1981).

Neste contexto, ainda se observam utilidade de estudos sobre relações hipsométricas em matas nativas, principalmente em áreas de cerrado. Por isso, o objetivo deste estudo foi avaliar o ajuste de modelos hipsométricos em um povoamento florestal inequiâneo, caracterizado como: fragmento de Cerrado Sensu stricto tocantinense.

\section{Material e métodos}

\section{Área de estudo e coleta de dados}

A área de estudo se localiza no sul do estado do Tocantins, no município de Gurupi, com coordenadas Latitude $11^{\circ} 43^{\prime}$ 45" S e Longitude 49 04' 07" W (FIGURA 1). A classificação climática de acordo com Köppen é Aw (clima tropical com estação seca no inverno) com precipitação pluvial anual em torno de $1.400 \mathrm{~mm}$. (SEPLAN, 2012). 
Figura 1. Localização da área de estudo

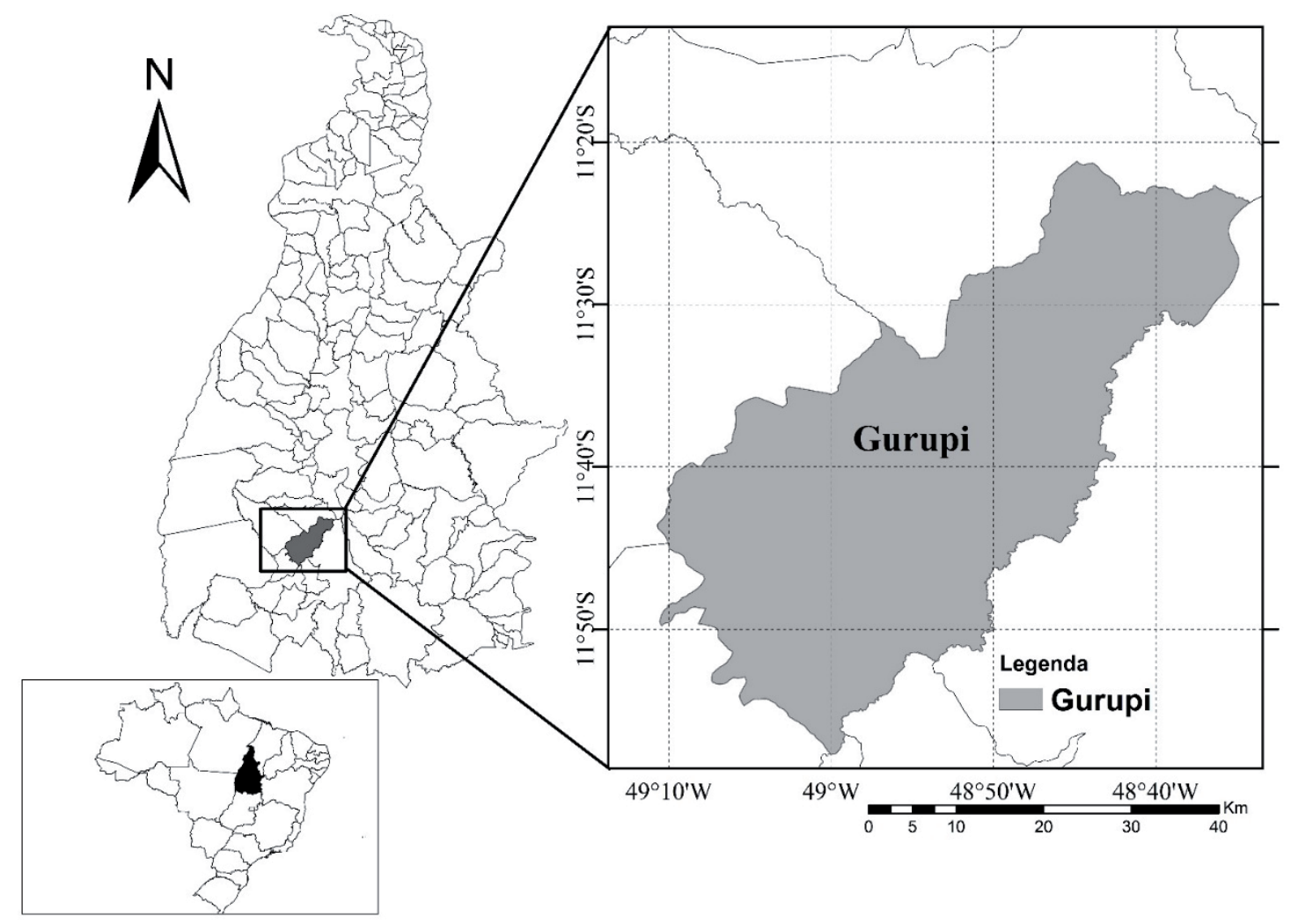

Fonte: Elaboração dos autores (2016)

O levantamento de campo foi realizado em um fragmento de Cerrado com cerca de 7,0 hectares, constituído de uma vegetação em processo de regeneração em estágio avançado. De acordo com levantamento histórico feito da área, concluiu-se que antigamente o local era de pastagens para atividade de pecuária.

Na coleta dos dados de altura total e circunferência a altura do peito (CAP), selecionou ao acaso três árvores mais próximas a um ponto amostral, medindo trinta árvores para uso no ajuste dos modelos hipsométricos; outras trinta árvores, com dados independentes, foram utilizadas na validação dos melhores modelos hipsométricos ajustados (FIGURA 2), avaliando-se, desta forma, a predição das alturas. O termo predição é utilizado para designar a altura obtida por meio de um modelo ajustado em que a árvore em questão não participou do seu ajuste (BATISTA et al, 2001).

Na medição das variáveis de diâmetro e altura, utilizou-se de fita métrica para mensurar o CAP (posteriormente foi transformado para diâmetro) e o hipsômetro Suunto, respectivamente. A opção pelo hipsômetro Suunto foi devido a este ser um instrumento bastante utilizado na área florestal (MAYRINCK et al. 2016; JESUS et al. 2012). 
Figura 2. Distribuição de pontos em que se mediram três árvores por ponto alocado aleatoriamente em 7,0 ha de Cerrado.

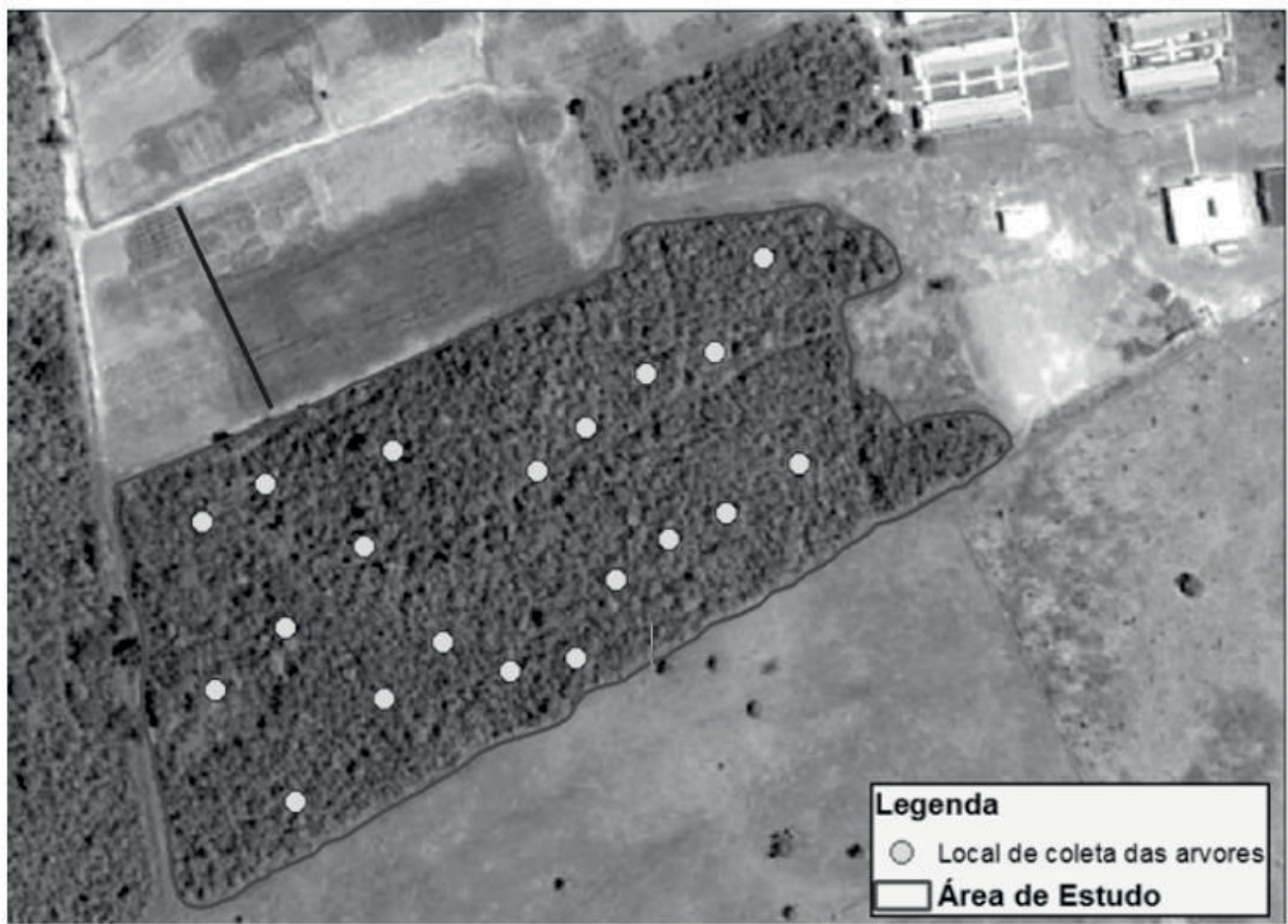

Fonte: Elaboração dos autores (2016).

\section{Modelos hipsométricos ajustados e critérios de avaliação}

Para os ajustes das relações hipsométricas, utilizaram-se dos modelos estatísticos apresentados na Tabela 1, os quais são comumente empregados para expressar a relação hipsométrica de árvores no Brasil (VENDRUSCOLO et al., 2015; SENA et al., 2015; CURTO et al., 2013). Esses modelos, cinco lineares e cinco não lineares, foram ajustados empregando-se o Software R For Windows considerando apenas o diâmetro como variável independente. 
Tabela 1. Modelos hipsométricos ajustados avaliados para um fragmento de Cerrado no sul do estado do Tocantins

\begin{tabular}{|c|c|c|}
\hline $\mathrm{N}^{\circ}$ & MODELOS & DENOMINAÇÃO \\
\hline \multicolumn{3}{|c|}{ LINEARES } \\
\hline 1 & $H=\beta 0+\beta 1 D+\beta 2 D^{2}+\varepsilon$ & PARABOLIC \\
\hline 2 & $\ln (H)=\beta 0+\beta 1 \ln (D)+\varepsilon$ & STOFFEL \\
\hline 3 & $\ln (H)=\beta 0+\beta 1(\underline{1})+\varepsilon$ & CURTIS \\
\hline 4 & $\mathrm{H}=\beta 0+\beta 1 \ln (\mathrm{D})+\varepsilon$ & HENRIKSEN \\
\hline 5 & & AZEVEDO \\
\hline \multicolumn{3}{|c|}{ NÃO LINEARES } \\
\hline 6 & $H=\beta 0(1-\beta 1 \exp (-\beta 2 D))+\varepsilon$ & MONOMOLECULAR \\
\hline 7 & $\mathrm{H}=\beta 0 \exp$ & GOMPERTZ \\
\hline 8 & $\mathrm{H}=\frac{\beta 0}{(1+\beta 1 \exp (-\beta 2 \mathrm{D}))}+\varepsilon$ & LOGISTIC \\
\hline 9 & $H=\beta 0\left(1-\exp \left(-\beta 1 D^{\wedge} \beta 2\right)\right)+\varepsilon$ & WEIBULL \\
\hline 10 & $H=\frac{D^{2}}{\left(\beta 0+\beta 1 D+\beta 2 D^{2}\right)}+\varepsilon$ & PRODAN \\
\hline
\end{tabular}

$\mathrm{H}=$ altura total; $\beta \mathrm{i}=$ parâmetros da regressão; $\mathrm{D}=$ diâmetro a altura do peito; $\mathrm{Ln}=$ logaritmo neperiano; Exp $=$ exponencial; $\varepsilon=$ erro.

Fonte: Elaboração dos autores (2016).

Em seguida, após o ajuste dos dez modelos hipsométricos, procedeu-se à avaliação preliminar desses por meio da análise de resíduos e das estatísticas obtidas a partir das Equações 1, 2 e 3, quais sejam as seguintes:

$$
\begin{gathered}
R^{2} a j=1-\left(\frac{n-1}{n-p}\right)\left(\frac{\text { sQres }}{s Q t}\right)(1) \\
S_{H A}=\sqrt{\frac{\text { SQres }}{\text { Glres }}}(2) \\
S_{H A} \%=\left(\frac{s_{H A}}{\dot{H}}\right) 100
\end{gathered}
$$

Em que:

SQres=Soma dos quadrados dos resíduos

$\mathrm{SQt}=$ Soma dos quadrados totais

$\mathrm{R}^{2} \mathrm{aj}=$ coeficiente de determinação ajustado

=erro padrão residual

=Média das alturas reais, demais já foram definidos.

Para definir o melhor modelo hipsométrico, foi adotado um valor ponderado dos escores dos parâmetros estatísticos (VP) para classificá-los conforme os resultados das estatísticas de ajuste, sendo o menor VP o indicador do melhor ajustamento. 
Na predição dos modelos previamente selecionados com os critérios já descritos anteriormente, adotou-se o erro absoluto (EA), média dos desvios do erro de predição (MDP), raiz quadrada do erro médio (RQEM\%) e coeficiente de determinação $\left(R^{2}\right)$, cujas estatísticas foram obtidas pelas Equações 4, 5, 6 e 7, quais sejam:

$$
\begin{aligned}
& E A=\sum|H-\hat{H}| \\
& M D P=\left(\frac{H-\hat{H}}{\frac{H}{n}}\right) 100 \\
& R Q E M \%=\left(\sqrt{\frac{\sum(H-\hat{H})^{2}}{n}}\right) 100 \\
& R^{2}=1-\left(\frac{\text { SQep }}{\text { SQt }}\right)
\end{aligned}
$$

altura predita

$\mathrm{n}=$ número de árvores

Sqep= soma dos quadrados dos resíduos de predição, demais já foram definidos.

\section{Resultados e discussões}

A Tabela 2 apresenta os resultados da estimativa dos dez modelos de relação hipsométrica ajustados na área de estudo deste trabalho. Nota-se que os modelos tiveram comportamento muito semelhantes entre si, com pouca variação nas estatísticas $R^{2} a j$ e $s_{H \hat{H}}$, em que o $R^{2}$ aj variou entre 0,362 (modelo 5) e 0,489 (modelo 7). Para $\mathrm{S}_{\mathrm{HH}}$, a variação foi entre 17,58 \% (modelo 7) e 20,29 $\%$ (modelo 3 ). Esses resultados de $\mathrm{R}^{2} \mathrm{aj}$ e $\mathrm{s}_{\mathrm{H} \hat{H}}$ podem ser considerados satisfatórios por se tratar de mata nativa de Cerrado, que apresentam alta variação na relação entre altura e diâmetro das árvores.

Isso implica baixa correlação dessas duas variáveis e, principalmente, valores de $\mathrm{R}^{2}$ aj não elevados, bem como o menor resultado das demais estatísticas quando comparado com o ajuste de modelos hipsométricos em floresta plantada (HESS et al. 2014). Em trabalhos sobre relação hipsométrica para plantios florestais, por exemplo, Azevedo et al. (2011), trabalhando com quatro espécies (sete-casca, ipê branco, ipê roxo e ipê amarelo) nativas no planalto da conquista na Bahia, encontraram em grande parte $\mathrm{R}^{2}$ aj superiores a $80 \%$.

Rufino et al. (2010), trabalhando com um povoamento de eucalipto conduzido pelo sistema de rebrota, obtiveram valores de $\mathrm{R}^{2}$ aj variando entre 0,55 e 0,76 \%. Por outro lado, Hess et al (2014) ajustaram modelos de relação hipsométrica para espécies da Floresta Amazônica e obtiveram $\mathrm{R}^{2} \mathrm{aj}$ variando desde 0,17 até o valor de 0,97 e entre 0,08 \% e 13,2\%. Geralmente, a complexidade do ajuste de modelos hipsométricos em mata nativa é bem maior que em plantios, principalmente em áreas de Cerrado (ANDRADE et al. 2015). 
Tabela 2. Estatísticas de ajuste de dez modelos hipsométricos testados em mata nativa de Cerrado

\begin{tabular}{ccccccc}
\hline Modelos & $\boldsymbol{\beta 0}$ & $\boldsymbol{\beta 1}$ & $\boldsymbol{\beta 2}$ & $\mathbf{s}_{\mathrm{HH}} \%$ & $\mathbf{R}^{\mathbf{2} a j}$ & $\mathbf{W V}$ \\
\hline 1 & 5,882 & 0,246 & $-0,0018$ & 17,88 & 0,470 & 10 \\
2 & 1,5071 & 0,2617 & - & 17,76 & 0,459 & 8 \\
3 & 2,347 & $-1,868$ & - & 20,29 & 0,295 & 23 \\
4 & 3,091 & 2,339 & - & 18,46 & 0,436 & 17 \\
5 & 1,039 & $-2,018$ & - & 19,30 & 0,362 & 17 \\
6 & $-1,5663$ & 0.7566 & 0,0632 & 17,90 & 0,451 & 20 \\
7 & 18,5930 & 0,6845 & 0,0199 & 17,58 & 0,489 & 5 \\
8 & 489,851 & 0,009 & 0,286 & 18,09 & 0,444 & 25 \\
9 & 15,8434 & 0,9781 & 0,0386 & 17,87 & 0,471 & 15 \\
10 & 14,6869 & 1,4350 & 0,0577 & 17,86 & 0,472 & 10 \\
\hline
\end{tabular}

$\beta$ 0, $\beta 1$ e $\beta$ 2: coeficientes de regressão; $S_{H \hat{H}}$ : Erro Padrão da Estimativa percentual $(\%) ; R^{2} a j:$ Coeficiente de Determinação ajustado; VP: Valor Ponderado dos Escores dos parâmetros estatísticos.

Fonte: Elaboração dos autores (2016).

Quando comparados entre si, os cincos modelos não lineares são muito semelhantes em termos de qualidade de ajuste. Observa-se que os modelos não lineares (TABELA 2), em geral, tiveram melhores resultados, destaque para o modelo $7 \mathrm{com} \mathrm{R} \mathrm{R}^{2}$ aj de 0,489 e $\mathrm{s}_{\mathrm{HH}}$ de 17,5 \%, resultando em menor valor de VP (5 pontos). Os modelos não lineares mostram-se menos sensíveis às particularidades apresentadas em áreas nativas, pois o padrão da curva altura-diâmetro é fortemente ligado pelas características biológicas que, nesse caso, foi melhor representado pelo modelo biomatemático de Gompertz (MODELO 7). Portanto, dos modelos ajustados, preliminarmente, os modelos 1, 2, 6, 7, 9 e 10 apresentaram-se de melhor desempenho sendo previamente selecionados.

Nas Figuras 3 e 4, são apresentados os gráficos da distribuição dos resíduos dos modelos previamente selecionados. Pode-se observar que as distribuições tiveram comportamento semelhante entre os modelos 1 e 2 (modelos lineares), com valores residuais enquadrando-se entre 30 e $-40 \%$ e com a maior parte dos pontos concentrando-se entre 20 e -20\%. Para os modelos 6, 7, 9 e 10 (modelos não lineares), os valores variaram entre 25 e -30\%, tendo uma distribuição bem uniforme nesse intervalo, demonstrando-se superiores aos lineares. Em relação ao erro metro, observa-se uma variação de dois metros, para mais e para menos, para todos os modelos. Sena et al. (2015) recomendam os modelos Logístico e Gompertz para estabelecimento de uma relação hipsométrica, sobretudo pela melhor distribuição de resíduos. 
Figura 3. Resíduos percentuais em função DAP $(\mathrm{cm})$ para seis modelos hipsométricos previamente selecionados em um fragmento de Cerrado no Tocantins.

Modelo 1

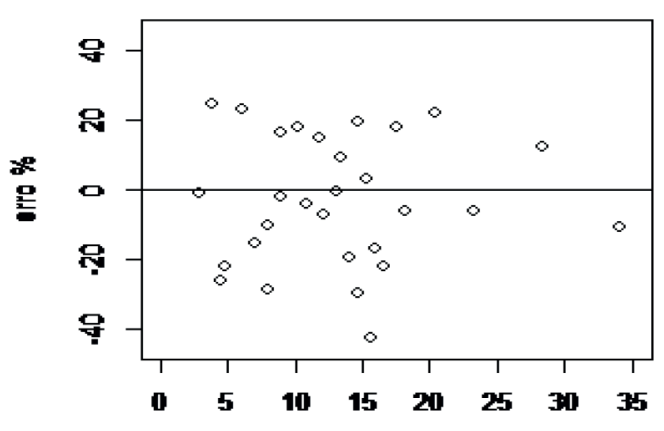

DAP

Modelo 6

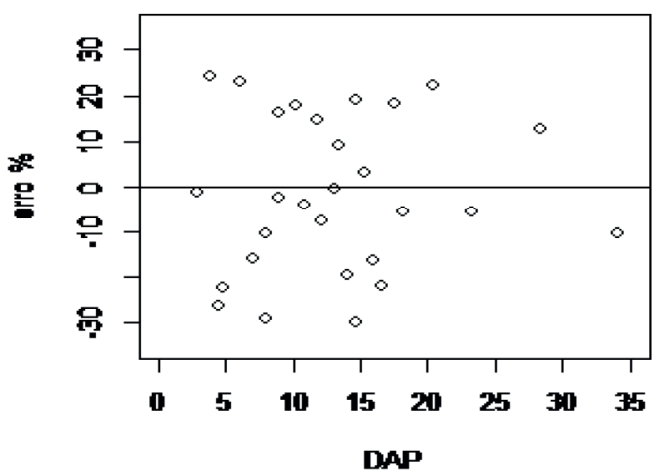

Modelo 9

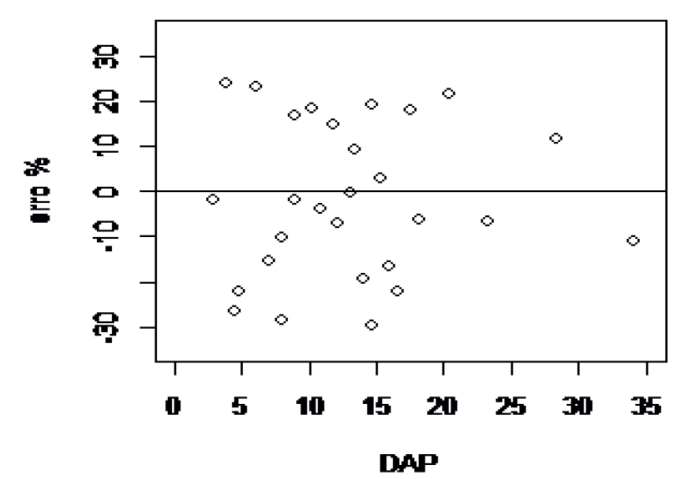

Modelo 2

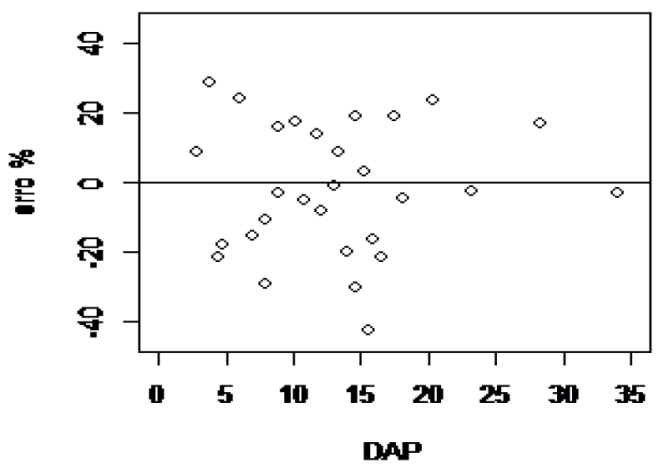

Modelo 7

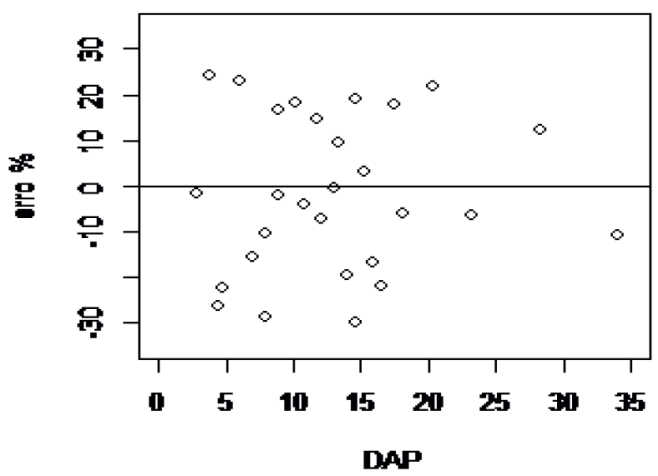

Todelo 10

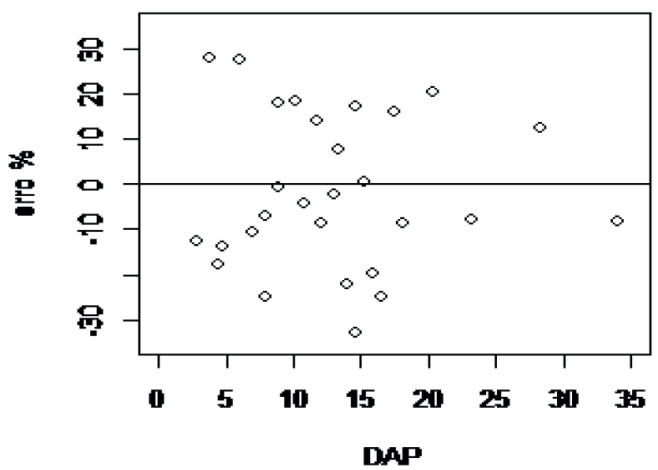

Fonte: Elaboração dos autores (2016). 
Figura 4. Distribuição de resíduos em relação à altura estimada para seis modelos hipsométricos previamente selecionados em um fragmento de Cerrado no Tocantins
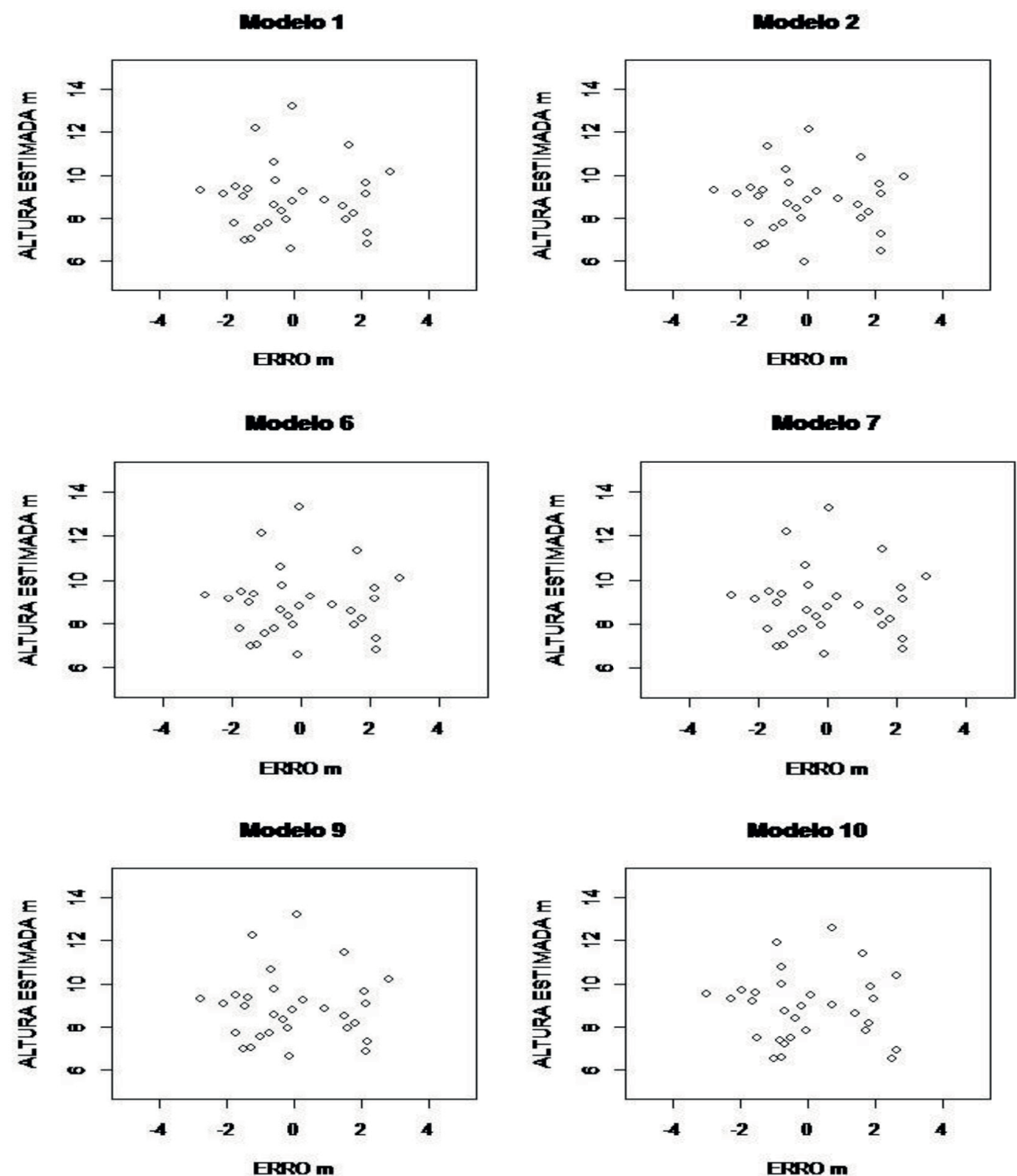

Fonte: Elaboração dos autores (2016).

Na Tabela 3 estão os resultados das estatísticas obtidas na predição feita com os seis modelos previamente selecionados. Nota-se que houve pouca variação nos critérios estatísticos de predição, sendo que os modelos 2 e 10 obtiveram os melhores valores, sendo mais favorável ao modelo 10 . Entre esses modelos, apesar de uma distribuição de resíduos variando de $20 \%$ à $-60 \%$, em geral, superestimando a altura para ambos modelos (FIGURA 5), nota-se um comportamento melhor para o modelo 10 mesmo sendo pouco expressivo. 
Tabela 3. Predição dos modelos com os melhores resultados de $\mathrm{R}^{2}$, Syx\% e gráfico de dispersão

\begin{tabular}{ccccc}
\hline Model & AE & ADP\% & SRAE\% & $\mathbf{R}^{2}$ \\
\hline 1 & 32,376 & $-13,725$ & 18,30 & 0,472 \\
2 & 31,455 & $-12,676$ & 17,69 & 0,507 \\
6 & 32,576 & $-13,798$ & 18,38 & 0,468 \\
7 & 32,436 & $-13,757$ & 18,33 & 0,471 \\
9 & 32,376 & $-13,663$ & 18,30 & 0,473 \\
10 & 28,701 & $-11,553$ & 16,45 & 0,574 \\
\hline
\end{tabular}

EA: erro absoluto; MDP: média dos desvios do erro em porcentagem; RQEM\%: raiz quadrada do erro médio; $\mathrm{R}^{2}$ : coeficiente determinação.

Fonte: Elaboração dos autores (2016).

Figura 5. Resíduos percentuais em função DAP $(\mathrm{cm})$ para os modelos hipsométricos 2 e 10 obtidos na predição da altura de árvores de um fragmento de Cerrado tocantinense.
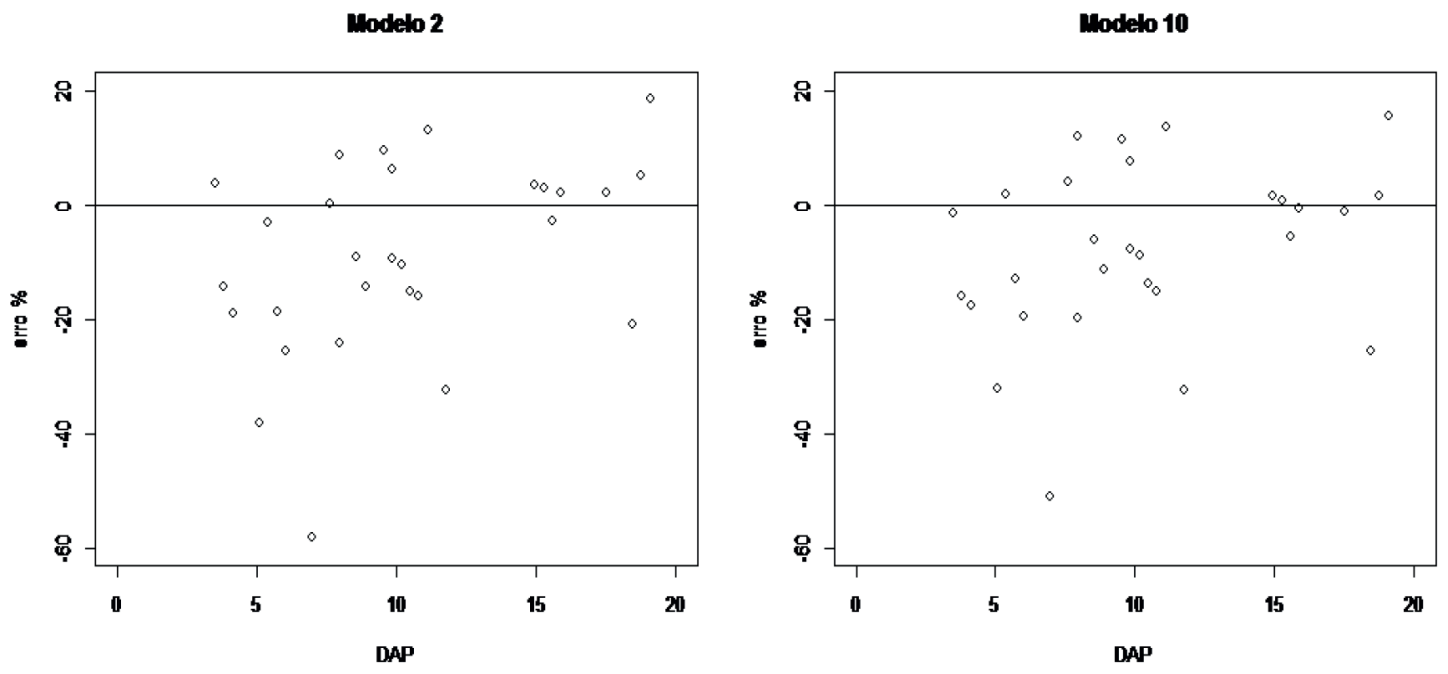

Fonte: Elaboração dos autores (2016).

Batista et al. (2001), avaliando a estabilidade de modelos hipsométricos, encontraram em uma área de mata nativa, floresta tropical, modelos lineares e não lineares estimando a altura preditiva com $R^{2}$ variando de 0,28 e 0,36 e RQEM de 9,8\% a 80,9\%, com superioridade dos modelos não lineares, mostrando que a fundamentação biológica da forma funcional dos modelos é essencial para que possam apresentar estimativas adequadas de altura. Resultado coerente com o deste trabalho.

Contudo, mesmo que os modelos lineares sejam mais simples, recomendam-se os modelos não lineares, pela sua capacidade de melhor explicar a relação altura e diâmetro das árvores, além da possibilidade de interpretação biológica dos seus parâmetros. 


\title{
Conclusão
}

Os valores estatísticos de ajuste dos modelos variaram entre si, $\mathrm{R}^{2} \mathrm{aj}: 0,362$ a 0,489 e $\mathrm{s}_{\mathrm{HH}}$ : $17,58 \%$ a $20,29 \%$. Na análise residual, observou-se uma tendência dos modelos de superesti-mar a altura, chegando até $40 \%$.

De forma geral, os modelos não lineares explicaram melhor a variável dependente altura quando comparados com os modelos lineares, sendo os mais recomendados para estimativa de alturas em áreas florestais de Cerrado.

Com a realização da predição, o melhor modelo hipsométrico foi o Prodan (não linear), que obteve melhores valores estatísticos e variação gráfica.

\section{Hypsometric models adjusted for a fragment of Sensu stricto Cerrado in Tocantins, Brazil}

\begin{abstract}
Hypsometric models are of great value in order to reduce forest inventory costs. The present work aimed to adjust and evaluate models of hypsometric relation at a Cerrado area in regenerative process, located in the municipality of Gurupi (TO). Ten models, including five linear and five non-linear, were tested. The adjustments were made through $\mathrm{R}$ software, for Windows. As criterion for the adjustment of models, the following statistical parameters were used: adjusted coefficient of determination ( $\left.\mathrm{R}^{2} \mathrm{aj}.\right)$, the residual standard error $\left(\mathrm{s}_{\mathrm{HH}}\right)$ and the graphical analysis of the residuals; then, the validation of the best adjusted models took place. The best adjustment statistics were for the non-linear models, with emphasis on Gompertz model (MODEL 7), R²aj: 0,489 and $\mathrm{s}_{\mathrm{HH}} 17.58 \%$.

The models, in general, overestimated the height, reaching up to $40 \%$ more than the real one. As a result, six models were referred for validation: two linear and four non-linear. The best model with prediction was the non-linear Prodan (model 10), which presented better statistical values and graphical variation. It is concluded non-linear models explained more satisfactorily the height-dependent variable.
\end{abstract}

Keywords: Tree height. Selection. Validation.

\section{Referências}

ANDRADE, V. C. L.; KROETZ, E. A.; NICOLA, A.; SOUZA, P. B.; NOHAMA, F. K.; LEITE, H. G.; BINOTI, D. H. B.; BINOTI, M. L. M. S. Amostragem e agrupamento de dados de relação hipsométrica em inventários florestais de Cerrado Tocantinense. Pesquisa Florestal Brasileira, Colombo, v. 35, n. 83, p. 227-238, 2015. Disponível em: <https://pfb.cnpf.embrapa.br/pfb/index.php/pfb/article/ view/683/430>. Acesso em: 26 nov. 2016.

ANDRADE, V. C. L.; MARTINS, S. V.; CALEGARIO, N.; CHICHORRO, J. F. Influência de três sistemas de amostragem na estimativa da relação hipsométrica e do volume de árvores em um fragmento de Mata Atlântica. Scientia Forestalis, Piracicaba, n. 70, p. 31-37, 2006. Disponível em: <https:// www.ipef.br/publicacoes/scientia/nr70/cap03.pdf>. Acesso em: 22 nov. 2016. 
AZEVEDO, G. B.; SOUSA, G. T. O.; SILVA, H. F.; BARRETO, P. A. B.; NOVAES, A. B. Seleção de modelos hipsométricos para quatro espécies florestais nativas em plantio misto no planalto da conquista na Bahia. Enciclopédia biosfera, Goiânia, v. 7, n.12, 2011. Disponível em: <http://www.conhecer. org.br/enciclop/2011a/agrarias/selecao\%20de\%20modelos.pdf>. Acesso em: 22 nov. 2016.

BATISTA, J. L. F.; COUTO, H. T. Z. do; MARQUESINI, M. Desempenho de modelos de relações hipsométricas: estudo de três tipos de floresta. Scientia Forestalis, n. 60, p.149- 163, 2001. Disponível em: <https://docplayer.com.br/51781023-Desempenho-de-modelos-de-relacoes-hipsometricas-estudo-em-tres-tipos-de-floresta.html>. Acesso em: 26 nov. 2016.

CURTO R. A.; SILVA G. F.; SOARES C. P. B.; MARTINS L. T.; DAVID H. C. Métodos de estimação de altura de árvores em floresta estacional semidecidual. Floresta, Curitiba-PR, v. 43, n. 1, p. 105-116, 2013. Disponível em: <https://revistas.ufpr.br/floresta/article/view/26791>. Acesso em: 24 nov. 2016.

CRECHE, E. H. Efeitos da densidade da plantação sobre a produção, crescimento e sobrevivência de Araucaria angustifólia (Bert.) 0. Ktze. em Missiones, Argentina. 1996. 172 f. Dissertação (Mestrado em Manejo Florestal) - Universidade Federal do Paraná, Curitiba, PR, 1996. Disponível em: <https://acervodigital.ufpr.br/handle/1884/25418>. Acesso em: 26 nov. 2016.

DRAPER, N. M.; SMITH, H. Applied Regression Analysis. 2. ed., New York, Wiley. 1981, 709 p.

HESS, A. F.; BRAZ, E. M.; THAINES, F.; MATTOS, P. P. Ajuste de relação hipsométrica para espécies da Floresta Amazônica. Ambiência, v. 10, n. 1 p. 21 - 29, 2014. Disponível em: <https://www. embrapa.br/florestas/busca-de-publicacoes/-/publicacao/989738/ajuste-de-relacao-hipsometrica-para-especies-da-floresta-amazonica>. Acesso em: 26 nov. 2016.

INOCÊNCIO, M. E.; CALAÇA M. Estado e território no Brasil: reflexões a partir da agricultura no Cerrado. Revista IDeAS, v. 4, n. 2, p. 271-306, 2010. Disponível em: <https://r1.ufrrj.br/cpda/ ideas/ojs/index.php/ideas/article/view/46>. Acesso em: 26 nov. 2016.

INSTITUTO BRASILEIRO DE GEOGRAFIA E ESTATÍSTICA (IBGE). Biomas do Brasil. Disponível em: <http://www.ibge.gov.br>. Acesso em: 10 jan. 2017.

JESUS. C. M.; MIGUEL, E. P.; LEAL, F. A.; ENCINAS, J. I. Avaliação de diferentes hipsômetros para medição da altura total em um povoamento clonal de Eucalyptus urophylla x Eucalyptus grandis. Enciclopédia biosfera, Goiânia, v. 8, n. 15, p. 291, 2012. Disponível em: <http://repositorio.unb.br/ handle/10482/16218 >. Acesso em: 24 nov. 2016.

MAYRINCK, R. C.; OLIVEIRA, X. M.; SILVA, G. C. C.; VITOR, P. C. G.; FERRAZ FILHO, A. C. Avaliação de hipsômetros e operadores na mensuração de árvores de Eucalyptus urograndis de tamanhos diferentes. Revista Verde de Agroecologia e Desenvolvimento Sustentável, Pombal, v. 11, n. 5, p. 9094, 2016. Disponível em: <https://www.gvaa.com.br/revista/index.php/RVADS/article/view/3976>. Acesso em: 26 nov. 2016.

PONTES NETO, T. P. Comparação de modelos lineares e não lineares em relações hipsômetricas para clone de Eucalyptus spp. no polo gesseiro do Araripe-Pe. Dissertação (Pós-Graduação em 
Ciências Florestais) - Universidade Federal do Rural do Pernambuco, Departamento de Ciências Florestais, Recife, 2012. Disponível em: <http:/www.ppgcf.ufrpe.br/sites/www.ppgcf.ufrpe.br/files/ documentos/tarcisio_pio_pontes_neto.pdf>. Acesso em: 26 nov. 2016.

RODRIGUES L. G. S. M.; RODRIGUES F. M. Inventário florestal de ambiente de Cerrado utilizando quatro técnicas de levantamento florestal. Enciclopédia Biosfera, Centro Cientifico Conhecer, v. 8, n. 15; p. 11-15, 2012. Disponível em: <http://www.conhecer.org.br/enciclop/2012b/ciencias\%20 agrarias/inventario\%20florestal.pdf>. Acesso em: 24 nov. 2016.

RUFINO, R. F.; MIGUEL, E. P.; SANTOS, G. A.; SANTOS, T. E. B.; SOUZA, F. Ajuste de modelos hipsométricos para um povoamento de eucalipto conduzido sobre o sistema de rebrota. Enciclopédia biosfera, Goiânia, v. 6, n. 10, 2010. Disponível em: <http://www.conhecer.org.br/enciclop/2010b/ ajuste.pdf>. Acesso em: 26 nov. 2016.

SANQUETTA, C. R.; CORTE, A. P. D.; ROGLIN, A.; PIMENTEL, A. Relações diâmetro-altura para espécies lenhosas em um fragmento de floresta Ombrófila Mista no Sul do Paraná. Iheringia, Porto Alegre, v. 68, n. 1, p. 103-114, 2013. Disponível em: <https://isb.emnuvens.com.br/iheringia/article/view/42>. Acesso em: 26 nov. 2016.

SCHNEIDER, P. R.; SCHNEIDER, P. S. P.; SOUZA, C. A. M de. Análise de regressão aplicada à Engenharia Florestal. Santa Maria: UFSM/CEPEF, 1998. 294p.

SENA, A. L. M.; SILVA NETO, A. J. da; OLIVEIRA, G. M. V.; CALEGARIO, N. Modelos lineares e não lineares com uso de covariantes para relação hipsométrica de duas espécies de pinus tropicais. Ciência Florestal, Santa Maria, v. 25, n. 4, p. 969-980, 2015. Disponível em: <https://periodicos.ufsm. br/cienciaflorestal/article/view/20651 > . Acesso em: 26 nov. 2016.

SECRETÁRIA DO PLANEJAMENTO E MEIO AMBIENTE (SEPLAN). Atlas do Tocantins: subsídios ao planejamento à gestão territorial. 6. ed. Palmas: 2012. p.18-21. Disponível em: <http://www.sefaz.to.gov.br/zoneamento/atlas-do-tocantins/>. Acesso em: 20 dez. 2016.

SOARES, C. P. B.; PAULA NETO, F. de; SOUZA, A. L. de. Dendrometria e inventário florestal. 2. ed. Viçosa: UFV, 2011. 272 p.

VENDRUSCOLO, D. G. S.; DRESCHER, R.; SOUZA, H. S.; MOURA, J. P. V. M.; MAMORÉ, F. M. D.; SIQUEIRA, T. A. S. Estimativa da altura de eucalipto por meio de regressão não linear e redes neurais artificiais. Revista Brasileira Biomas, São Paulo, v. 33, n. 4, p. 556-569, 2015. Disponível em: <http://jaguar.fcav.unesp.br/RME/fasciculos/v33/v33_n4/A9_DiogoRonaldo.pdf>. Acesso em: 24 nov. 2016.

\section{Histórico editorial}

Submetido em: 23/02/2017

Aceito em: 26/12/2017 